ISSN: 2162-3104 Print/ ISSN: 2166-3750 Online

Volume 8, Issue 2 (2018), pp. 1152-1072

(C) Journal of International Students

http://jistudents.org/

doi: 10.5281/zenodo. 1250417

\title{
Cultural Identification, Acculturation, and Academic Experience Abroad: A Case of a Joint Faculty-Led Short-Term Study Abroad Program
}

\author{
Jeongyi Lee \\ Kennesaw State University, USA \\ Kathryn Negrelli \\ Kennesaw State University, USA
}

\begin{abstract}
The number of students expanding their academic programs to include learning experiences abroad has been steadily increasing over the past two decades. This study investigates the cultural identification of short-term study abroad participants and its influence on their acculturation process. By means of a widely used acculturation scale, it was found that there were statistically significant effects of cultural experiences on cultural identification, specifically decreases in cultural identification scores. Implications from this study suggest a deeper understanding of students' ethnic identifications can be helpful in developing study abroad programs that promote the goals of student awareness, cultural sensitivity, and global competence.
\end{abstract}

Keywords: acculturation, cultural identification, orthogonal cultural identification scale, study abroad, intercultural competence

There is no doubt that we are living in an era of globalization, in a world in which nearly every industry demands a global perspective to be successful. Resources point to the resulting increase in the number of students electing study abroad (hereafter, SA) as part of their academic experience (Institute 
for International Education, 2016; OECD, 2009). With a projected increase in student global mobility in the coming decade, educators today are charged with the tasks of better understanding those concepts that are ultimately related to enhancing intercultural learning and minimizing cultural differences. This trend has led to a growing body of research in the areas of cultural identity, which includes one's beliefs, values, ethnicity, social practices, etc. Cultural identity plays a role in influencing one's process of identifying with another group, as people will bring with them their practices, values, and traditions to the host country or cultures with which they become associated. The interwoven and inseparable nature between cultural identity and identification has been observed in studies, mainly in the fields of health, behavioral sciences and social sciences (Angstman, Harris, Golbeck \& Swaney, 2009; Campesino, Belyea, \& Schwartz, 2009; Howarth, Wagner, Magnusson, \& Sammut, 2014; Lawton \& Gerdes, 2014). However, investigation into students' cultural identification and acculturation within the SA context is scarce. Furthermore, numerous studies have shown the benefits of SA experiences, yet suggest that these benefits are limited and inconsistent across programs.

Therefore, this research investigated the cultural identification of students on a short-term SA program and its influence on their intercultural sensitivity and acculturation process. The purpose of this study was to measure the level of cultural identification of SA participants before and after their intercultural interactions in order to discover the effects of those interactions on their cultural identifications and ultimately their acculturation processes. The overarching goal is to pursue the development of study abroad programs that promote intercultural competence for students in global learning contact situations. The theoretical background is grounded in Oetting and Beauvais's (1990-1991) Orthogonal Cultural Identification Theory, which suggests that cultural identification dimensions are independent of each other, implying that an increasing identification with one culture does not demand a decreasing identification with another. Guiding the study were the following research questions:

- Is there a significant change in students' cultural identification following participation in our SA program?

- Is there an increase in Asian ethnic identification with increased interaction with Asian culture? 
The next section reviews literature on SA programs and on acculturation, specifically the acculturation measurement scale employed in this study. We then discuss our methodology and findings, concluding with a discussion and implications for future research.

\section{LITERATURE REVIEW}

This research investigates the relationships between cultural identifications of students on a SA program and the influences of such relationships on their acculturation process. This section begins with a review of literature on the larger topic of SA programs, moves into studies of identification and cultural identity, and merges these areas with the theme of intercultural competence and sensitivity developed through learners' acculturation processes experienced on SA programs. It concludes with a review of various studies using the ethnic identification scale employed in this investigation.

\section{Study Abroad and Cultural Identity/Identification}

SA programs in higher education have been evolving and growing, more rapidly in the past two decades, as an outcome of the surge of institutions to internationalize and produce global citizens. These programs are often used as recruitment tools for students who look beyond academic offerings to opportunities to experience international education. University websites often highlight the importance placed on SA programs, and help young adults understand that studying abroad and having intercultural experiences is no longer a luxury, but rather a necessity for them to compete in the global market.

"University of Georgia is dedicated to preparing you to become a global citizen equipped to respond to the changes and challenges that affect our local and global communities." (UGA, Office of International Education).

"Study abroad programs...foster the development of...crucial intellectual skills, and the benefits span far beyond the workplace. Becoming a global citizen will transform the way you see the world in a way that makes you highly desirable to potential employers, while deeply enriching your personal life." (NIU, Study Abroad).

The increase in SA programs has brought about a number of research studies, many of which aim to identify key factors that lead to developing intercultural competence. These studies investigate such areas as 
what and how students learn in a SA context (Dunkley, 2009; Vande Berg, 2007), the critical element of pre- and post- management of SA programs (Chinnappan, McKenzie, \& Fitzsimmons, 2013; Santoro \& Major, 2012), service learning or community engagement within a SA program (Crabtree, 2013; Pusch \& Merrill, 2008), and the impact duration of the program has on the learning outcomes of the SA participants (Anderson, Lawton, Rexeisen, \& Hubbard, 2006; Campbell \& Walta, 2015; Hamad \& Lee, 2013).

Concerning what length of SA program has most positive outcomes, research results are mixed. An overwhelming number of studies that include Dwyer (2004), Kehl and Morris (2007-2008), Medina-Lopez-Portillo (2004), and Kehl and Morris (2007-2008) have produced findings that support conventional wisdom: the longer the SA program, the more significant the students' academic, cultural development and personal growth. Yet there has been a significant increase in the number of shorter programs offered (i.e., less than one academic year) and in the number of students enrolling in them, and researchers are finding that these programs also have a positive impact on cross cultural development and intercultural competence (Anderson, et al., 2006; Chieffo \& Griffiths, 2004; DonnellySmith, 2009). Other studies on short term programs have led to new knowledge in areas such as how the type of program and destination have an effect on global citizenship as seen through volunteerism (Horn \& Fry, 2013), and how program length is associated with both changes in cultural identification (Hamad \& Lee, 2013) as well as in the cross-cultural adaptation process (Kim, 2001).

It is clear that SA journeys expose students to the reality of cultural differences and the possibility of challenges to their sense of being. As such, a number of research studies have investigated the influence of self-concept and cultural identity on SA experiences and the degree of interaction with the host culture as factors of the degree of intercultural effectiveness or success of one's acculturation while abroad. Peng, Van Dyne, and Oh (2015) in their study of undergraduate business students on a short-term SA, found that the beneficial effects of "motivational CQ", or cultural IQ as defined by Ang \& Van Dyne (2008) and Earley \& Ang (2003), a concept closely related to intercultural competence, is contingent upon the strength of an individual's cultural identity, defined as the degree to which one identifies with his/her own national culture. The interconnectedness of identity and its influence on the SA journey is also illustrated in Gieser's 
(2015) study of American SA students' approaches to and negotiations of their encounters with various sociocultural practices at a university in South Africa, and the role their cultural identities played in the sense-making process. In his interviews with the American subjects, nearly every one raised the issue of their American identity impacting their SA experiences, yet whether that issue actually benefited them or not depended on the individual. These findings are furthered by Mayer's (2009) study on the relationship between identity, conflict and its management in the work context, which finds how a person reacts to cultural and group conflicts, and in a broader sense, challenges in the differences in culture in a SA experience, and that this depends largely on the strength of their cultural identity.

Research has shown varying degrees to which identity, ethnic values, beliefs, and practices affect the acculturation process of SA students. In this study, the definition of acculturation is adopted from Berry (2006): "the dual process and cultural and psychological change that takes place as a result of contact between two or more cultural groups and their individual members" (p.13). A brief review of the literature on the scale measuring acculturation and self-identification used in this study are examined below.

\section{Acculturation Measurement}

Methods of measurement in acculturation research typically fall into the general categories of unidimensional (adapting to the mainstream cultural context at the expense of one's heritage tradition, or assimilation), bidimensional (maintaining one's heritage cultural tradition and adopting the new mainstream cultural tradition in two separate and independent processes), and multidimensional (adding another dimension to the bidimensional method in order to account for participants who traverse two or more cultural heritage traditions and mix cultural elements into hybrids). This current study employed the Orthogonal Cultural Identification Scale (hereafter, OCIS), as posited by Oetting and Beauvais (1990-1991), which asserts that individuals may identify with more than one culture without necessarily sacrificing one cultural identity for another. Stated simply, cultural identification dimensions are independent of each other. Past investigations using the OCIS are reviewed below. 


\section{Orthogonal Cultural Identification Theory}

The theory behind orthogonal cultural identification and its measurement scale, developed by Eugene Oetting and Fred Beauvais (19901991), acknowledges the influence of a pluralistic environment and normalized experiences of identification with multiple cultures, and reflects the dynamic and fluid nature of cultural identification. This six-item instrument includes survey questions about family way of life, personal way of life, family success, personal success, family cultural traditions, and personal cultural traditions. Identification with any culture can be assessed, as long as the culturally related items on the scale are carefully constructed so as to be familiar to those taking the test. A total score can be created for each individual culture and then combined or used separately.

The orthogonal cultural identification theory and OCIS have made significant contributions to research in the fields of health, behavioral sciences and social sciences. In one study, Angstman et al. (2009), used OCIS to investigate the relationship between cultural identification and smoking among American Indian (AI) adults. They found 1) that high AI identification predicted smoker status and high reservation, 2) orthogonal identification status did not predict smoker status when controlling for age and reservation residence, and 3) positive responses to certain individual OCIS items predicted smoker status when controlling for age and reservation residence. Based on their data, they concluded that among some groups of urban AIs, recreational smoking is associated with AI cultural identification. Their findings lead toward an implementation and evaluation of interventions that support AI cultural identification as a way to reduce alcohol and drug abuse among AIs, specifically adolescents.

In Campesino et al.'s (2009) study of spirituality among Latino and non-Latino College students, the OCIS was used in conjunction with the Latino Spiritual Perspective Scale. They found that 1) Latinos scored significantly higher than non-Latinos in both measures of spiritual perspectives, and 2) behavioral measures, such as frequency of personal prayer, were also higher among the Latino group. Findings from their study indicate that spirituality among Latinos has meanings specific to the cultural group context. Such conclusions have implications for nursing research involving the conceptualization and measurement of spirituality among multiethnic groups.

Oetting and Beauvais's (1990-1991) investigation into the cultural identification of Native-American and Mexican-American youth showed 
that (1) identification with Anglo (White American) culture is related to having Anglo friends and to family acceptance of an Anglo marriage, (2) there is a degree of personal and social strength in identifying with either the minority or the majority culture, and (3) this strength does not necessarily translate into less drug use, because drug use is related to how much the culture that the person identifies with approves or disapproves of drugs. Similarly, Strunin and Demissie's (2001) study employed the OCIS to assess cultural identification in their study of alcohol use among AfricanAmerican and Haitian Black adolescents. Their conclusion was that there was no relationship between cultural identification and alcohol use, and that youths in both groups reported drinking alone due to stress and the attempt to relax. Their findings indicate that sociocultural factors, context and the basis of drinking are issues that warrant attention in programs for youth.

Many studies that report using the OCIS to investigate cultural identification deal with such issues of substance use, family, and self-esteem in the fields of health, behavioral sciences and social sciences. However, little has been researched in the area of SA and the role cultural identification plays in successful SA programs and student acculturation. The next section explains the methods of this present study, which includes the assessment of cultural identification via the OCIS.

\section{RESEARCH METHOD}

\section{Research Context}

This study was based on a sample of university students participating in a semester-long SA program beginning in January 2016, culminating in a 24-day trip to Japan and Korea (12 days in each country) in May-June 2016. The objectives of the program were to explore contrasts of traditional and modern cultures of both countries through observations and interactions with Japanese and Korean people and society. The intense credit-bearing program included requirements of extensive readings, internet researching, film viewings, online and in-class discussions, quizzes, and service learning project preparation conducted in four 4-hour pre-departure meetings. In addition, students were required to maintain a daily journal throughout the trip. Upon return, they participated in a 2-hour post-trip meeting, and submitted two final projects. 


\section{Participants}

The participants in the study were 17 students at a large university in the Southeast U.S., enrolled in a short-term SA program to Japan and Korea in the spring of 2016. All participants provided a signed consent form, approved by the university Institutional Review Board. There were 12 females and 5 males with a mean age of 21.4 years and with various majors. Thirteen participants were born in the U.S. The four foreign-born subjects came to the U.S. at the ages of 8, 4, 2 and before 1 year, and all had been living in the U.S. for at least 16 years. None had ever traveled either to Japan or Korea, with the exception of one Japanese heritage student who once traveled in Japan for 2 months since she left Japan at the age of 8, and another student who traveled in Korea for 1 day. In terms of their first language, 14 of the subjects listed English and three listed Spanish. Regarding their parents' language and birthplace, four Spanish-speaking parents were born in Mexico, Costa Rica, and El Salvador, Japanesespeaking parents were born in Japan, and Cantonese-speaking parents were born in Vietnam and Malaysia. Considering this background information, participants were grouped into White and Non-white for a summative variable of ethnicity. Information concerning their previous experience with the target languages of Japanese and Korean was gleaned from their responses to their self-assessed proficiency of the target languages. Participants were then divided into two distinctive groups - those with experience and those with none-for further descriptive statistics in terms of their previous experience with the target languages.

\section{Instrument}

The OCIS, designed for cross-cultural use, was used to measure subjects' ethnic and cultural identification. This empirically validated instrument measures identification with five American ethnic groups (Asian, Anglo, Latino, African, and Native American). The term "ethnic group" refers to broad groupings of Americans on the basis of both race and culture (Oetting \& Beauvais, 1990-1991). The six base questions of OCIS incorporating attitudes and behaviors include items on family way of life, personal way of life, family success, personal success, family cultural traditions, and personal cultural traditions. Each set of questions was asked separately for different cultures. It did not, therefore, focus on specific culture-related behaviors but on general attitudes toward involvement in a culture. Respondents used a 4-point scale from 1 (none at all) to 4 (a lot) to 
answer each item, and separate scores for each ethnicity were averaged from the six base questions. High identification with a given ethnicity is indicated by a score of 3 or more, medium identification by a score of 2, and low identification by a score of 1 or less. A high score on OCIS indicates a strong identification with the culture. In addition to this acculturation measure, all participants completed a demographic questionnaire that served to provide their background information.

\section{Procedure}

All acculturation questionnaires were self-administered online by the subjects twice: (1) immediately prior to the beginning of the SA coursework in January, to assess their initial level of acculturation (pre-test); and (2) upon return from their 24-day SA program in June to assess their exit level of acculturation (post-test). The demographic questionnaire was administered once in January. At the completion of the survey, the responses were scored, with 1 through 4 assigned to a total of 102 response entries where 1 was associated with the least identification with the culture ("none at all"). These data were then imported into SPSS for statistical analyses. Although the limited amount of data did not permit the use of many statistical tests for significance, the data were then subjected to a number of inferential (probability-based) statistics.

\section{RESULTS}

\section{Cultural Identification of SA Participants}

Based on the participants' responses to their ethnic and language backgrounds in the demographic questionnaire, the ethnicity variable was classified into White and Non-white and previous experiences with Korean and Japanese variables into With Experience and No Experience for further descriptive statistics. Table 1 displays the distribution of participants in terms of the four background variables.

Participants exhibited various combinations of cultural identification, such as a single identity, a bicultural identity, a high identification with one culture and a modest one with another, and a weak identification with all cultures. However, as Table 2 presents, inspection of cell means revealed that there was a slight decline in the level of cultural identification with each of the six cultures after the SA program. 
Table 1. Distribution of SA Participants

\begin{tabular}{llcc}
\hline & & $\mathrm{N}$ & Percent \\
\hline Gender & Male & 5 & 29.4 \\
& Female & 12 & 70.6 \\
Ethnicity & White & 9 & 52.9 \\
& Non-White & 8 & 47.1 \\
Korean & No Experience & 8 & 47.1 \\
\multirow{2}{*}{ Japanese } & With Experience & 9 & 52.9 \\
& No Experience & 5 & 29.4 \\
& With Experience & 12 & 70.6
\end{tabular}

Note. Means and standard deviations for the six composite groups of preand post-OCIS scores appear in Table 2 .

Table 2. Means and Standard Deviations for Six OCIS Group Measures

\begin{tabular}{lcccc}
\hline & \multicolumn{2}{c}{ Pre } & \multicolumn{2}{c}{ Post } \\
& $\mathrm{M}$ & $\mathrm{SD}$ & $\mathrm{M}$ & $\mathrm{SD}$ \\
\hline White & 3.22 & .91 & 2.97 & .87 \\
Asian & 2.10 & 1.11 & 1.83 & .98 \\
Mexican & 2.11 & 1.25 & 1.86 & 1.20 \\
Black & 1.81 & .99 & 1.68 & 1.01 \\
Indian & 1.53 & .81 & 1.34 & .74 \\
Other & 1.56 & .94 & 1.44 & .74 \\
Total & 2.05 & & 1.86 & \\
\hline
\end{tabular}

In relation to the purpose of studying the set of cultural identification groups, the basic questions are whether there is a relationship between cultural identification groups, and, if a relationship in fact exists, how strong or weak that relationship is. Therefore, as a preliminary step to conducting analyses of the effects of demographic personal variables on both pre- and post-OCIS scales, correlations among the six composite cultural identification groups were calculated in order to determine the degree of collinearity among these groups. These correlations are reported in 
Tables 3 and 4. The conventional alpha level of .05 was used as the criterion for statistical significance. The results of the correlational analyses of preOCIS scores presented in Table 3 show that in all cases except for the White group, correlations of all the other groups with each other were statistically significant at the .01 level, and all correlations were positive. All the correlations of the cultural groups on post-OCIS showed the same pattern of relationships as those of pre-OCIS. Moreover, the identification with White culture and that with Native American culture were moderately and positively correlated with each other at the .01 level.

Table 3. Correlations among the six Groups on Pre-test

\begin{tabular}{lllllll}
\hline & White & Asian & Mexican & Black & Indian & Other \\
\hline White & 1.00 & .110 & -.032 & .124 & $.231^{* *}$ & .112 \\
Asian & & 1.00 & $.343^{* *}$ & $.418^{* *}$ & $.534^{* *}$ & $.625^{* *}$ \\
Mexican & & & 1.00 & $.266^{* *}$ & $.388^{* *}$ & $.548^{* *}$ \\
Black & & & & 1.00 & $.511^{* *}$ & $.447^{* *}$ \\
Indian & & & & & 1.00 & $.755^{* *}$ \\
Other & & & & & & 1.00 \\
\hline
\end{tabular}

**. Correlation is significant at the 0.01 level. $\mathrm{N}=17$

Table 4. Correlations among the Six Groups on Post-test

\begin{tabular}{lllllll}
\hline & White & Asian & Mexican & Black & Indian & Other \\
\hline White & 1.00 & .145 & .110 & -.164 & -.063 & .066 \\
Asian & & 1.00 & $.419^{* *}$ & $.313^{* *}$ & $.472^{* *}$ & $.582^{* *}$ \\
Mexican & & & 1.00 & $.352^{* *}$ & $.336^{* *}$ & $.420^{* *}$ \\
Black & & & & 1.00 & $.521^{* *}$ & $.410^{* *}$ \\
Indian & & & & & 1.00 & $.692^{* *}$ \\
Other & & & & & & 1.00 \\
\hline
\end{tabular}

**. Correlation is significant at the 0.01 level. $\mathrm{N}=17$

\section{Relationship between Pre- and Post-OCIS Measures}

By looking at the mean difference between pre- and post-OCIS scores for each cultural identification group shown in Table 2 to determine which set of scores is higher or lower and how much they differ, it was found that all the post-OCIS scores were lower than the pre-OCIS scores. A univariate mixed factorial 6 (ethnic group) x 2 (pre-test, post-test) ANOVA on the OCIS scores showed a significant difference $(\mathrm{p}<.0001)$ among the six cultural identification groups as rated by all participants. There was also 
a significant difference $(\mathrm{p}<.05)$ between the pre-test and post-test. However, the tests indicated that there was no significant interaction between the two tests (pre vs. post) and the cultural identification groups. In order to determine if these differences in the mean scores were statistically significant, a paired sample $t$ test comparing means was conducted. The results of the paired sample $t$ test revealed that the mean difference between the pre-test $(\mathrm{M}=3.22, \mathrm{SD}=0.91)$ and post-test scores $(\mathrm{M}=2.97, \mathrm{SD}=$ 0.87) of students' identification with White American culture was statistically different, $\mathrm{t}(101)=2.453, \mathrm{p}<.01$; the mean difference between the pre-test $(\mathrm{M}=2.10, \mathrm{SD}=1.11)$ and post-test scores $(\mathrm{M}=1.83, \mathrm{SD}=$ 0.98) of students' identification with Asian American culture was statistically different, $\mathrm{t}(101)=2.636, \mathrm{p}<.005$; the mean difference between the pre-test $(\mathrm{M}=2.11, \mathrm{SD}=1.25)$ and post-test scores $(\mathrm{M}=1.86, \mathrm{SD}=$ 1.20) of students' identification with Mexican American culture was statistically different, $\mathrm{t}(101)=2.554, \mathrm{p}<.01$; and the mean difference between the pre-test $(\mathrm{M}=1.53, \mathrm{SD}=0.81)$ and post-test scores $(\mathrm{M}=1.34$, $\mathrm{SD}=0.74)$ of students' identification with American Indian culture was statistically different, $\mathrm{t}(101)=2.206, \mathrm{p}<.05$.

\section{Pre- and Post-OCIS by Participant Background}

The population means for the scores on the pre- and post-OCIS differed for participants from different backgrounds: (1) gender (Male and Female); (2) ethnicity (White and Non-white); (3) past experience with Korean (No Korean and Korean); and (4) past experience with Japanese (No Japanese and Japanese). Figures 1-4, descriptive presentations of interactions as well as cell means of both pre- and post-OCIS scores for all four personal variables, indicate some interesting patterns.

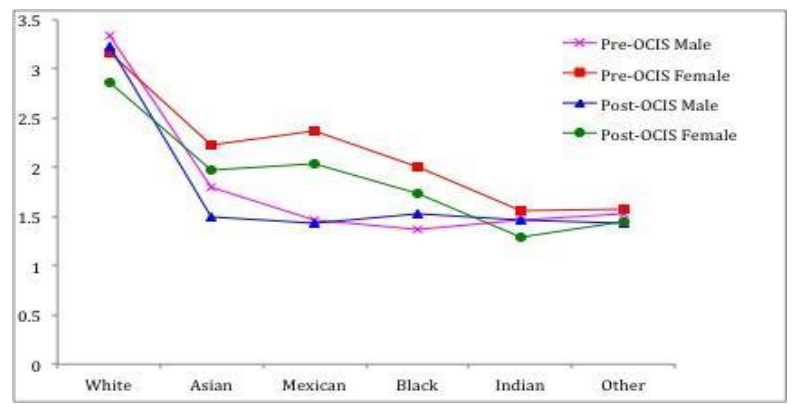

Figure 1. Interactions of gender and cell means of cultural identification on pre- and post-OCIS 


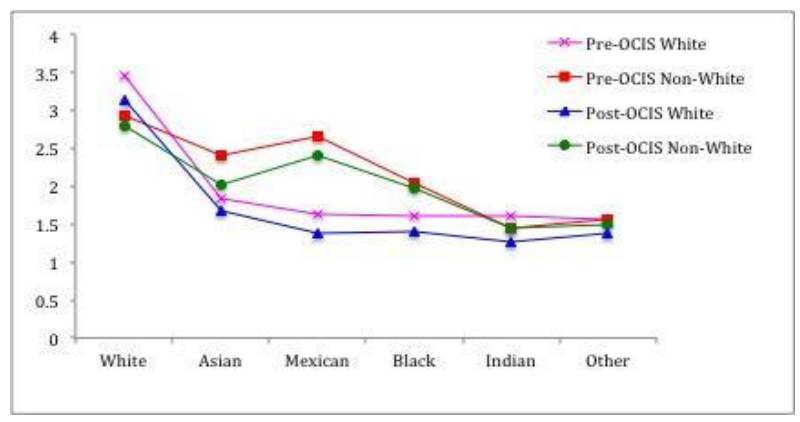

Figure 2. Interactions of ethnicity and cell means of cultural identification on pre- and post-OCIS

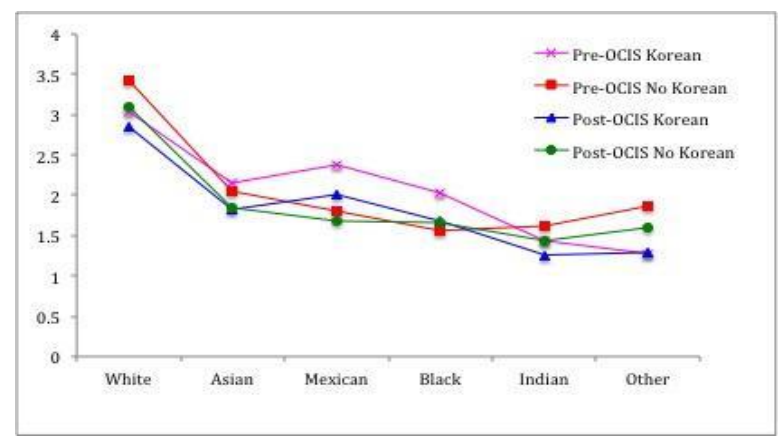

Figure 3. Interactions of Korean experience and cell means of cultural identification on pre- and post-OCIS

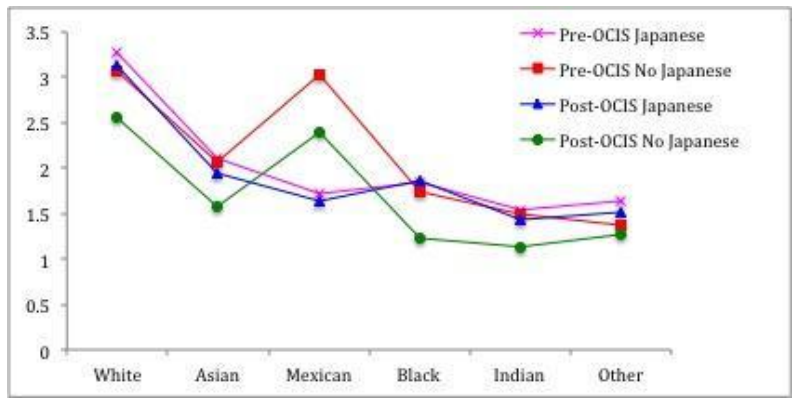

Figure 4. Interactions of Japanese experience and cell means of cultural identification on pre- and post-OCIS 


\section{CONCLUSIONS AND DISCUSSIONS}

The OCIS questionnaire, in conjunction with a demographic questionnaire, was used to assess levels of cultural identification of 17 American university students before and after participating in a SA program to Japan and Korea. The paired sample t test conducted to evaluate whether a difference existed between the cultural identification scores before and after the SA program indicated that there was a decrease in students' cultural identification with each of the six cultures following their participation in the program, and that the decreases in the cultural identification scores of White American, Asian American, Mexican American, and American Indian cultures were statistically significant. Thus, intercultural interaction during SA did correlate-negatively - with cultural identification scores. It was hypothesized that scores of cultural identification with Asian culture on post-OCIS would show an increase from the pre-OCIS scores. As the paired sample t test revealed, however, there was a statistically significant decrease in students' cultural identification with the Asian American culture following their participation in our SA program, where they had both implicit and explicit interactions with the Asian culture.

\section{Discussion}

The changes in the cultural identification scores over time in this study support Oetting and Beauvais's (1990-1991) argument that cultural identification is a social learning process and the result of the interaction between the individual and his or her environment. The fundamental premise of their theory-that cultural identification dimensions are independent of each other-is also supported by our findings as increases in identification scores with one culture did not result in subsequent decreasing identification scores with another culture.

In this study, we measured American college students' cultural identifications before and after their immersion in two societies, Japan and Korea, during a short-term SA program, reflecting degrees of effects of new experiences. Results of this study provide an index of the degree of immersion of students in their cultural identifications, illuminating how ethnic identification and acculturation are related within a SA experience. The findings showed statistically significant decreases in scores of cultural identification with White American, Asian American, Mexican American, 
and American Indian cultures after the completion of the program. What might be plausible explanations for these decreases in scores?

Our SA participants were in transition throughout the course of the program, and they moved in the path of the transition across cultural identification space. Although most participants continued to maintain a reasonably high level of cultural identification with one or more cultures in the path of the transition, there seemed to be a loss of overall identification, which might likely have been accompanied or caused by stress, as claimed by Oetting (1993). It is not unlikely that the challenges of traversing different cultures and negotiating different sets of values, norms and identities, as one does when experiencing a different country and culture, generate a certain level of stress. Travelling to not one but two distinctly different countries and cultures may have compounded the stress and anxiety levels for our SA participants. Not only were they expected to navigate differences in food, customs, and communication, and participate in cultural and social activities outside of their familiar environments, but they also had to contend with the within-group pressures of living, cooperating, and sharing daily experiences among themselves for 24 continuous days. Additionally, there were the demands and rigid requirements of the SA program pre-departure, in-country, and upon return to the U.S. The factors of pressure and stress may have created adjustment challenges, constantly occurring experiences that are a normal part of the adaptation of both the culture and the individual, and may have contributed to the unexpected decreases in cultural identification scores that resulted in this study. According to Oetting (1993: 38), people who demonstrate a decline of cultural identification "test their limits, get confused, construct inaccurate cognitions, deviate from cultural norms, try new roles, and grow and change." Our participants throughout the course of the program experienced the same. The resulting decline in cultural identification, however, should not be construed as an adverse or harmful phenomenon as there was no evidence of negative attitudes towards the cultures developed.

Duration of the SA program might be also a factor in the decrease of participants' cultural identification scores. Past research has shown that longer SA programs result in more significant students' academic, cultural development and personal growth (e.g., Dwyer, 2004; Hamad \& Lee, 2013; Kehl \& Morris, 2007-2008; Medina-Lopez-Portillo, 2004) and this might be relevant to cultural identification as well. Perhaps more cultural activities and interactions with the target cultures for more prolonged periods of time 
may have produced an increase rather than decrease in the cultural identification scores as they relate to Asian cultures. Nevertheless, in spite of its brevity, the impact of our SA program on the participants' cultural identification was indeed powerful in that the outcomes were reflected in their view of their cultures and served as a catalyst in shaping their cultural identification.

\section{Implications for Acculturation and SA Program Development}

Acculturation processes can influence behavior. Acculturative changes have been documented in all the behavioral domains in this study to various extents, including family way of life, personal way of life, family success, personal success, family cultural traditions, and personal cultural traditions. In practice, however, the impact of acculturation on psychological and behavioral changes in students on SA programs is underestimated at best and ignored at worst. Thus, the quantitative assessment of cultural identification with regard to SA participants in this study contributes to the advancement of ethnic identity and acculturation study to capture more of the complexity inherent in cultural identification. Overall, this study supports the prevalent view in the field of acculturation that identification with any one culture is independent from identification with other cultures. Indeed, identification with any one culture was virtually a continuous dimension in this study. Interpreting identification in this innovative manner is relative to the typical dichotomous categorization of identification found in many acculturation studies. This identification process reiterates the importance of integrating a variety of relevant cultural components such as participation in traditional activities, cultural behaviors, feeling successful within a culture, and family involvement in cultural activities. Duration of the cultural experience also plays an important role in the identification process. The process can equally be applied to different cultural groups or communities in the U.S., such as SA groups to help to shape the cultural identification of participants and to provide insight into how to design and implement SA programs. This requires committed, sustained efforts from administrators as well as educators. Fundamental to these efforts is a clearer understanding of those who participate in the program and why they have chosen it as part of their academic, global experience. With an understanding of this fundamental background information, we are in a better position to create more meaningful curricula for students all across the cultural identification continuum. In short, the 
notion of cultural identification and acculturation relies heavily on the interaction of various factors and can be defined in a multivariate manner, as the present study illustrates.

\section{Limitations of the Study}

Although this study has covered new ground in the fields of acculturation and SA programs by investigating and documenting the SA participants' cultural identification process that is not often addressed empirically, it is not without its limitations. First, the assessment measures focused specifically on behavioral acculturation outcomes of a small, albeit diverse target group over a short-term program. Future research should consider a larger sample of participants to enable more sophisticated approaches to the measurement of acculturation, and conducted over a longer-term SA program. The data could be then subjected to a number of statistical tests for significance that will allow for an exploration of the relationship between demographic variables and cultural identification on acculturation. Second, although this study did include diverse ethnic groups, (i.e., White, Asian, Hispanic, and Black), the representations of each group was limited to a small number of subjects in which all ethnic subgroups were merged into one cohort group and compared with the majority ethnic group, White. It would, therefore, be beneficial for future studies to evaluate the acculturation process among other groups of diverse ethnicities as well. Third, this study relied heavily on self-rated questionnaires, and therefore is open to bias and inaccurate measurement of cultural identity and acculturation, both complex subjects. Future research could include more rigorously designed studies supplemented and augmented by findings from more comprehensive qualitative analyses.

\section{Conclusion}

Individual differences in cognitive, emotional, and behavioral determinants of acculturation are not highly researched. The present study of participants on a short-term SA program, which examined cultural identification as "a long-term underlying characteristic that organizes cognitions, emotions, and behaviors", as defined by Oetting (1993: 34), revealed that cultural identification indeed develops and is maintained through exchanges and interactions with others and with the cultural environment. The individual and collective interactions of our program participants ultimately brought about alterations in their cultural 
identifications and shaped and altered subsequent behaviors. The changes in post-OCIS scores were consistently lower across the cultures, supporting Oetting and Beauvais's principle of independence of cultural identification dimensions, and may be considered a transformation towards a more divergent cultural identification. Although maintaining aspects of their original culture while integrating other cultures may indeed be stressful and challenging, it can also be an adaptive approach that promotes cultural competency, mental health, and the well-being of individuals living in multicultural environments as well as the dynamic nature of society. And as the U.S. becomes increasingly multicultural and the era in which we live more globalized, this cultural competency is not only an asset but also a necessity.

\section{REFERENCES}

Anderson, P. H., Lawton, L., Rexeisen, R. J., \& Hubbard, A. C. (2006) Short-term study abroad and intercultural sensitivity: A pilot study. International Journal of Intercultural Relations, 30, 457-469.

Ang, S., \& Van Dyne, L. (2008). "Conceptualization of cultural intelligence: Definition, distinctiveness, and nomological network" in S. Ang, \& L. Van Dyne (Eds.), Handbook of cultural intelligence: Theory, measurement, and applications (pp. 3-15). Armonk, NY: M. E. Sharpe.

Angstman, S., Harris, K. J., Golbeck, A., \& Swaney, G. (2009). Cultural identification and smoking among American Indian adults in an urban setting. Ethnicity \& Health, 14(3), 289-302.

Berry, J. W. (2006) "Acculturation: A conceptual overview" in M. H. Bornstein \& L. R. Cote (Eds.), Acculturation and parent-child relationships Measurement and development (p.13): Mahwah, NJ: Lawrence Erlbaum Associates, Inc.

Campbell, W., \& Walta, C. (2015). Maximising intercultural learning in short term international placements: Findings associated with orientation programs, guided reflection and immersion. Australian Journal of Teacher Education, 40(10), 1-15.

Campesino, M., Belyea, M., \& Schwartz, G. (2009). Spirituality and cultural identification among Latino and non-Latino college students Hispanic Health Care International, 7(2), 72-79.

Chieffo, L. \& Griffiths, L. (2004). Large scale assessment of student attitudes after a short-term study abroad program. Frontiers: The Interdisciplinary Journal of Study Abroad, XVIII, 101-118.

Chinnappan, M., McKenzie, B., \& Fitzsimmons, P. (2013). Pre-service teachers' attitudes towards overseas professional experience: Implications for 
professional practice. Australian Journal of Teacher Education, 38(12), 36-54. DOI: $10.14221 /$ ajte.2013v38n12.5

Crabtree, R. D. (2013). The intended and unintended consequences of international service-learning. Journal of Higher Education Outreach and Engagement, 17(2), 43-65.

Donnelly-Smith, L. (2009). Global learning through short-term study abroad. Peer Review, 11(4), 12-15.

Dunkley, M. (2009). What students are actually learning abroad and how to improve the learning experience. Retrieved Nov. 29, 2016 from https://researchgate.net/publications228450410.

Dwyer, M. M. (2004). More is better: The impact of study abroad program duration. Frontiers: The Interdisciplinary Journal of Study Abroad, X, 151-163.

Earley, P. C., \& Ang, S. (2003). Cultural intelligence: Individual interactions across cultures. Stanford, CA: Stanford University Press.

Gieser, J. (2015). A sociocultural investigation of identity: How students navigate the study abroad experience. Journal of College Student Development, 56(6), 637-643.

Hamad, R., \& Lee, C. (2013). An assessment of how length of study-abroad programs influences cross-cultural adaptation. Journal of Human Behavior in the Social Environment, 23(5), 661-674.

Horn, A., \& Fry, G. (2013). Promoting global citizenship through study abroad: The influence of program destination, type, and duration on the propensity for development volunteerism. Voluntas: International Journal of Voluntary and Nonprofit Organizations, 24(4): 1159-1179. DOI 10.1007/s11266012-9304-y

Howarth, C., Wagner, W., Magnusson, N., \& Sammut, G. (2014). 'It's only other people who make me feel black': Acculturation, identity and agency in amulticultural community. Political Psychology, 35(1), 81-95.

Institute of International Education. (Nov. 14, 2016). Open doors. Retrieved Dec. 3, 2016 from http://www.iie.org/Who-We-Are/News-and-Events/PressCenter/Press-Releases/2016/2016-11-14-OpenDoorsData\#.WENeDyMrLLY.

Kehl, K., \& Morris, J. (2007-2008). Differences in global mindedness between short-term and semester-long study abroad participants at selected private universities. Frontiers: The Interdisciplinary Journal of Study Abroad, XV, $67-79$.

Kim, Y. Y. (2001). Becoming Intercultural. Thousand Oaks, CA: Sage.

Lawton, K.E., \& Gerdes, A.C. (2014). Acculturation and Latino Adolescent Mental Health: Integration of Individual, Environmental, and Family Influences. Clinical Child and Family Psychology Review, 17(4), 385-398.

Mayer, C. H. (2009). Managing conflicts through strength of identity. Management Revue, 20(3), 268-293. 
Medina-Lopez-Portillo, A. (2004). Intercultural learning assessment: The link between program duration and the development of intercultural sensitivity. Frontiers: The Interdisciplinary Journal of Study Abroad, X, 179-199.

Northern Illinois University, Study Abroad. "Why Study Abroad?” Retrieved April 16, 2017 from http://www.niu.edu/studyabroad/

OECD. (2009). Higher education to 2030. Vol.2, Globalization. Retrieved Dec. 3, 2016 from https://www.oecd.org/edu/ceri/44101074.pdf.

Oetting, E. R. (1993). Orthogonal cultural identification: Theoretical links between cultural identification and substance use. In M. R. De La Rosa \& J. L. Recio Adrados (Eds.), Drug abuse among minority youth: Advances in research and methodology (pp. 32-56), Rockville, MD: National Institute on Drug Abuse.

Oetting, E. R., \& Beauvais, F. (1990-1991). Orthogonal cultural identification theory: The cultural identification of minority adolescents. International Journal of the Addictions, 25(5), 655-685.

Peng, A. C., Van Dyne, L., \& Oh, K. (2015). The influence of motivational cultural intelligence on cultural effectiveness based on study abroad: The moderating role of participant's cultural identity. Journal of Management Education, 39(5), 572-596.

Pusch, M., \& Merrill, M. (2008). Reflection, reciprocity, responsibility, and committed relativism: Intercultural development through international service learning. In V. Savicki (Ed.), Developing intercultural competence and transformation: Theory, research, and application in international education (pp. 297-321). Sterling, VA: Stylus.

Santoro, N., \& Major, J. (2012). Learning to be a culturally responsive teacher through international study trips: transformation or tourism? Teaching Education, 23(3), 309-322.

Strunin, L., \& Demissie, S. (2001). Cultural identification and alcohol use among "Black" adolescents. Journal of Substance Use and Misuse, 36(14), 20252041.

University of Georgia, Office of International Education. "=About Education Abroad. Retrieved April 16, 2017 from https:/goabroad.uga.edu/ index.cfm?FuseAction=Abroad. ViewLink\&Link ID=8C651E86-5056BA1F-747BC7AEA57CA1C5

Vande Berg, M. (2007). Intervening in the learning of U.S. students abroad. Journal of Studies in International Education, 11(3/4), 392-399.

JEONGYI LEE, Ph.D., is an assistant professor of Korean. Her research interests lie in foreign/heritage language learners' motivation from SLA perspectives, topics of cultural/linguistic adaptation among U.S. immigrants, especially code-switching between L1 and L2 and speech perception of both Korean and English in L2 speakers of Korean in comparison with heritage 
speakers of Korean, and language learners' attitudes and motivations, and outcomes in study abroad programs. Email: jlee234@kennesaw.edu.

KATHRYN NEGRELLI, Ph.D., is an assistant professor of Japanese. Her research focuses on foreign language pedagogy, technology in the foreign language classroom, intercultural competence and acculturation. Email: knegrell@kennesaw.edu

Manuscript submitted: December 22, 2016 Manuscript revised: May 4, 2017 Accepted for publication: March 18, 2018 